\title{
Paraneoplastic autoimmune multi- organ syndrome and paraneoplastic pemphigus describe the same spectrum of disease pathology
}

\author{
Aimee S. Payne, Michael Kasperkiewicz, Christoph T. Ellebrecht, \\ Hayato Takahashi, Jun Yamagami, Detlef Zillikens and Masayuki Amagai
}

We appreciate the opportunity to discuss the characteristics of paraneoplastic pemphigus (PNP), further than was possible in our original Primer (Pemphigus. Nat. Rev. Dis. Primers 3, $17026(2017))^{1}$, afforded by Kyle T. Amber in his recent Correspondence (Paraneoplastic autoimmune multi-organ syndrome is a distinct entity from traditional pemphigus subtypes. Nat. Rev. Dis. Primers https://doi. org/10.1038/nrdp.2018.12 (2018)) $)^{2}$, which proposes classifying PNP as a pemphiguslike subtype of paraneoplastic autoimmune multi-organ syndrome (PAMS).

We agree that the PAMS terminology is more inclusive of the heterogeneous clinical presentation and pathophysiology of this disease than the term PNP, particularly in relation to the T cell-mediated cytotoxic effects associated with lichenoid tissue reactions, the extension of the humoral response to nondesmoglein autoantigens and the involvement of organs other than the skin. In the Primer, we use the term PNP to refer to the entire disease spectrum, whereas Amber proposes that the term PAMS, previously coined by Grando and colleagues ${ }^{3}$, is more appropriate. Both the Primer and the Correspondence highlight the aspects of PNP presentation, pathophysiology and prognosis that differ from classic pemphigus, including severe erosive stomatitis, bronchiolitis obliterans and high mortality. Thus, it is important that the lichenoid variant of PNP is recognized as a paraneoplastic process, so that underlying conditions and potential complications can be diagnosed and managed.

However, we disagree with the statement that PNP "is an unrelated condition" to pemphigus. Several studies have identified shared histological features and pathophysiological mechanisms of the bullous lesions in PNP and pemphigus vulgaris (PV): the skin blisters in PNP show suprabasal acantholysis characteristic of blisters observed in patients with PV, passive transfer of anti-desmoglein 3 antibodies isolated from patients with PNP to neonatal mice causes blisters that are histologically identical to those in patients with $\mathrm{PV}^{4}$ and anti-desmoglein $3 V_{\mathrm{H}}$ gene usage in one patient with PNP matched the immunodominant gene usage reported in patients with PV ${ }^{5,6}$. Thus, PNP and PV demonstrate common histological, pathophysiological and immunogenetic disease mechanisms.

The major limitation of our current knowledge of PNP is that we have not yet identified the scope of autoantigens recognized by $\mathrm{T}$ cells in the autoimmune cellular responses that lead to lichenoid tissue reactions. Once the autoimmune targets of those T cells are identified, we should have a better understanding of the pathophysiology of PNP in the skin and other organs. Of note, desmoglein 3-reactive T cells induce lichenoid tissue reactions in the skin as well as in the lung in mice, suggesting that PNP could be linked to PV through this shared target antigen both in cellular and humoral autoimmune reactions $\mathrm{s}^{7,8}$.

We propose that either term, PNP or PAMS, would be appropriate to describe this disease, and that it should be acknowledged that the term PNP is more precisely used to describe disease presentations that incorporate pemphigus-like bullous lesions, whereas PAMS is more inclusive to describe all disease presentations.

Aimee S. Payne ${ }^{1}$, Michael Kasperkiewicz², Christoph T. Ellebrecht ${ }^{1}$, Hayato Takahashi ${ }^{3}$, Jun Yamagami ${ }^{3}$, Detlef Zillikens ${ }^{2}$ and Masayuki Amagai ${ }^{3 *}$

'Department of Dermatology, University of Pennsylvania, Philadelphia, PA, USA.
${ }^{3}$ Department of Dermatology, Keio University School of Medicine Tokyo, Japan

*e-mail: amagai@med.keio.ac.jp doi: 10.1038/nrdp.2018.13 Published online 22 Feb 2018

1. Kasperkiewicz, M. et al. Pemphigus. Nat. Rev. Dis. Primers 3, 17026 (2017).

2. Amber, K. T. Paraneoplastic autoimmune multi-organ syndrome is a distinct entity from traditional pemphigus subtypes. Nat. Rev. Dis. Primers https://doi.org/10.1038/nrdp.2018.12 (2018).

3. Nguyen, V. T. et al. Classification, clinical manifestations, and immunopathological mechanisms of the epithelial variant of paraneoplastic autoimmune multiorgan syndrome: a reappraisal of paraneoplastic pemphigus. Arch. Dermatol. 137 193-206 (2001).

4. Amagai, M., Nishikawa, T., Nousari, H. C., Anhalt, G. J. $\&$ Hashimoto, T. Antibodies against desmoglein 3 (pemphigus vulgaris antigen) are present in sera from patients with paraneoplastic pemphigus and cause acantholysis in vivo in neonatal mice. J. Clin. Invest. 102, 775-782 (1998).

5. Saleh, M. A. et al. Pathogenic anti-desmoglein 3 mAbs cloned from a paraneoplastic pemphigus patient by phage display. J. Invest. Dermatol. 132, 1141-1148 (2012).

6. Cho, M. J. et al. Shared VH1-46 gene usage by pemphigus vulgaris autoantibodies indicates common humoral immune responses among patients. Nat. Commun. 5, 4167 (2014)

7. Takahashi, H. et al. Desmoglein 3-specific CD4 + $\mathrm{T}$ cells induce pemphigus vulgaris and interface dermatitis in mice. J. Clin. Invest. 121, 3677-3688 (2011).

8. Hata, T. et al. Ectopic expression of epidermal antigens renders the lung a target organ in paraneoplastic pemphigus. J. Immunol. 191, 83-90 (2013).

\section{Competing interests}

A.S.P. has previously served as a consultant for Syntimmune and TC Therapeutics and receives or has received grants or research support from the US NIH (R01-AR057001, R56-AR064220 and R01-068288), the Dermatology Foundation and Sanofi. She is co-inventor on a patent related to chimeric immunoreceptor therapy of pemphigus. The content is solely the responsibility of the authors and does not necessarily represent the official views of the US NIH. C.T.E. has received grants or research support from Deutsche Forschungsgemeinschaft (EL711/1-1) and is co-inventor on a patent related to chimeric immunoreceptor therapy of pemphigus. H.T. receives grants from Japan Society for the Promotion of Science. D.Z. is an advisory board member for Roche Pharma, and a consultant for Euroimmun, Almirall, UCB, Fresenius and arGEN-X. He received speakers honoraria and/or travel/accommodations/meeting compensation from Biotest, Fresenius, Miltenyi, Roche Pharma, Biogen Idec, AbbVie, UCB, Janssen and grants or research support from Euroimmun, Miltenyi, Fresenius, Biotest, Dompe, Almirall, Biogen and Roche. He holds the patent Euroimmun: DE 10 2006059574 A1. M.A. has received speaker honoraria and grants from Nihon Pharmaceutical, research support from Medical \& Biological Laboratories, Health Sciences Research Grants for Research on Rare and Intractable Diseases from Ministry of Health, Labour, and Welfare, and grants from Japan Society for the Promotion of Science and Agency for Medical Research and Development. M.K. and J.Y. declare no competing interests.

How to cite this article

Payne A.S. et al. Authors' reply: Paraneoplastic autoimmune multi-organ syndrome and paraneoplastic pemphigus describe the same spectrum of disease pathology. Nat. Rev. Dis. Primers 4, 18013 (2018) 